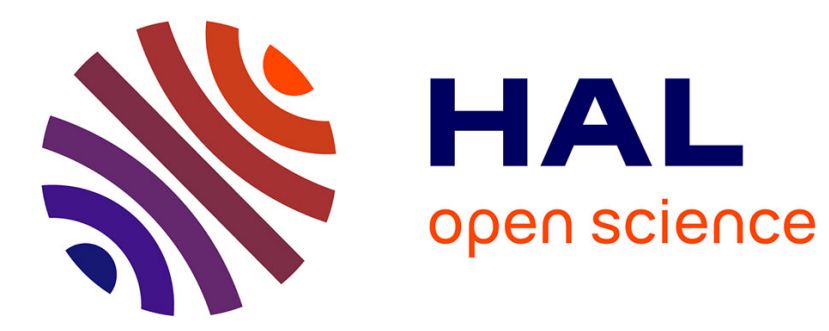

\title{
On the analytical and numerical simulation of an oscillating drop in zero-gravity
}

\author{
A. Aalilija, Ch.-A. Gandin, Elie Hachem
}

\section{To cite this version:}

A. Aalilija, Ch.-A. Gandin, Elie Hachem. On the analytical and numerical simulation of an oscillating drop in zero-gravity. Computers and Fluids, 2020, 197, pp.104362. 10.1016/j.compfluid.2019.104362 . hal-02428686

\section{HAL Id: hal-02428686 \\ https://hal.science/hal-02428686}

Submitted on 3 Jan 2021

HAL is a multi-disciplinary open access archive for the deposit and dissemination of scientific research documents, whether they are published or not. The documents may come from teaching and research institutions in France or abroad, or from public or private research centers.
L'archive ouverte pluridisciplinaire HAL, est destinée au dépôt et à la diffusion de documents scientifiques de niveau recherche, publiés ou non, émanant des établissements d'enseignement et de recherche français ou étrangers, des laboratoires publics ou privés. 


\title{
On the analytical and numerical simulation of an oscillating drop in zero-gravity
}

\author{
A. Aalilija, Ch.-A. Gandin, E. Hachem \\ MINES ParisTech, PSL - Research University, Centre for material forming (CEMEF), CNRS UMR 7635, CS 10207, 1 rue \\ Claude Daunesse, 06904 Sophia Antipolis Cedex, France
}

\begin{abstract}
The oscillation of a levitated drop is a widely used technique for the measurement of the surface tension and viscosity of liquids. Analyses are mainly based on theories developed in the nineteenth century for surface tension driven oscillations of a spherical, force-free, liquid drop. However, a complete analysis with both analytical and numerical approaches to study the damped oscillations of a viscous liquid drop remains challenging. We first propose in this work an extension of the theory that includes the coupled effects of surface tension and viscosity. The analytical solution permits derivation of both properties simultaneously, which is of interest for fluid with unknown viscosity. Then, the robustness of an Eulerian framework to simulate the fluid flow is discussed. Simulations of different oscillations modes for a liquid iron droplet immersed in a low density gas and comparisons with the derived theory are detailed and presented.
\end{abstract}

Keywords: Fluid flow, surface tension, numerical simulation, Level-Set method, oscillating drop method.

\section{Introduction}

Surface oscillation of liquid drops surrounded by a low density fluid (e.g., gas) is a classical problem in fluid mechanics. It can be observed in a wide range of practical applications such as in inkjet printing [1], fuel atomization process [2], ripple formation during spot welding [3], powder production by gas atomization [4] and containerless processing of levitated drops [5]. One of the main physical phenomena behind the oscillation of fluid drops is surface tension. It is well-known that when a liquid drop is sustained in a gas under microgravity, the surface tension makes the drop minimise the area of its interface with air. The equilibrium shape which provides the minimal area is a sphere. When the drop is distorted from its equilibrium shape, it displays oscillations with a frequency correlated to surface tension. If the liquid is viscous, the oscillations are damped with a decay rate linked to viscosity. The knowledge of how the observed frequency and the damping rate are related to surface tension and viscosity yields the access to the values of these properties. This is the principle of the oscillating drop technique used for the measurement of surface tension and viscosity [6].

Shape oscillation of drops driven by surface tension has been addressed theoretically by several authors over more than a century. The earliest theoretical investigation started with Rayleigh who derived, under the irrotational flow assumption, the frequency spectrum of small-amplitude axisymmetrical oscillations of non-viscous drops in vacuum with zero gravity [7]. This analysis was extended by Lamb to inviscid drops immersed in an inviscid medium [8]. Lamb also showed that for weakly viscous liquids the frequency

Email addresses: ayoub.aalilija@mines-paristech.fr (A. Aalilija), charles-andre.gandin@mines-paristech.fr (Ch.-A. Gandin), elie.hachem@mines-paristech.fr (E. Hachem) 
spectrum is identical to that found by Rayleigh and the assumption of the irrotational flow can be used to determine the damping rate [9]. Valentine et al. used the same approximation to derive the frequency and the damping rate of oscillating drops in liquid-liquid systems [10]. Miller and Scriven established that the irrotational approximation does not account for the dissipation in the boundary layer near the interface which is predominant for liquid-liquid systems even though the viscosities are very low, unless the droplet size is very small [11]. They proposed a general solution for the linear oscillations of liquid droplets hosted in another fluid medium for arbitrary viscosities including the interfacial viscoelastic properties. However, their solution is limited to free non-extensible interfaces. Prosperetti derived a more general solution [12] and showed that for the limiting case of low viscosities the drop behaves as a damped harmonic oscillator [13]. Further studies of this problem analyzed the non-linear oscillations starting from moderate-amplitudes by Tsamopoulos and Brown [14] to large-amplitudes with Lundgreen and Mansour [15], Trinh and Wang [16], and Foote [17]. A correction of the Rayleigh theory that includes the effect of both temperature variations and large-amplitudes was also proposed by Xiao et al. [18].

A number of other investigations have been dedicated to the oscillations of a drop sustained against gravity by a levitation technique. The use of a strong levitator field can make the equilibrium shape of the levitated drop aspherical. Thus, the presence of the levitator field affects the dynamics of the drop interface and the theories cited above must be revisited. For this purpose, Cummings and Blackburn proposed an approximated solution for a non-viscous drop in an electromagnetic levitator [19]. Later on, their work was extended by Bratz and Egry to account for the viscous dissipation effect for the same levitation technique [20]. However, their theories cannot take into consideration the electromagnetic stirring which leads to turbulent flow in the bulk liquid. In the presence of turbulence, the observed damping rate includes the effect of the turbulent viscosity which is not modelled in the available theories.

For sake of more accuracy, measurements under microgravity is carried out, preferentially within the limit of maximum $1 \%$ small-amplitude axisymmetrical oscillations with mode $n=2[5]$. The objective is to approach at best the approximations of the Rayleigh and Lamb theories. Despite these efforts, it remains difficult to tailor experimental conditions. The thorough interpretations of raw data is indeed challenging due to various deleterious effects: oscillation amplitude, sample rotation and procession, temperature variation, mixture of oscillation modes [14]-[18]. Only very recently and based on careful analyses of selected experimental data conducted in the international space station, Wunderlich and Mohr concluded that non-linear effects are not present even when reaching up to $10 \%$ deformation in liquid metallic drops [21].

Most of these theoretical analyses confine the attention to three-dimensional (3D) cases regarding their relevance for experiments and real applications whereas the two-dimensional (2D) cases have been marginally addressed. The 2D oscillations of a free-surface around a circular shape correspond to the oscillations occurring in a transverse section of a liquid jet injected from a non-circular orifice and showing no longitudinal variations. This configuration has been studied by Rayleigh for an inviscid liquid [7]. For the best of our knowledge, no analytical solution for damped oscillations of this $2 \mathrm{D}$ configuration has been explicitly derived. This configuration will be simply referred to as the "2D drop" case. The present work is an extension of Rayleigh's theory by adding the viscous effect of the inner flow. Lamb addressed the damped oscillations for small viscosities assuming a priori that the oscillation frequency found by Rayleigh is not altered by viscosity. Unlike Lamb, the solution derived hereafter applies for whatever value of the viscosity, i.e. for finite viscous and potential forces. Furthermore, it will be shown that three possible regimes of an initially-distorted drop can describe its behaviour: aperiodic regime, critically-damped regime and oscillatory regime. In the latter regime, the oscillation frequency results from the interaction between surface tension and viscosity. Although the effect of the non-negligible viscosity has been studied by Prosperetti in more comprehensive and complex cases[12]-[13], the present work re-derives the 3D solution in a rather simple way through the energy balance. The novelty lies also in the 2D solution which can provide a quantitative benchmark for testing the accuracy and the robustness of numerical modelling of multiphase flows.

Moreover, despite the maturity of the numerical modelling of the flows in two fluids separated by an interface, further efforts are still required to cope with the increasing demand of accuracy to study the damped oscillations of a droplet. Many numerical challenges can be encountered, especially when the 
properties of the two fluids abruptly change at the interface and interfacial forces are dominating [22]. The present work starts from the mathematical problem of the damped oscillations for $2 \mathrm{D}$ and $3 \mathrm{D}$ drops. The analytical solution will be derived for the time-evolution of the radius of the free-surface as well as the spatial-temporal variation of the velocity field in the bulk liquid. A numerical framework completes these developments in order to solve the unsteady Navier-Stokes equations for incompressible two-fluid flows. Simulations for different oscillations modes are then performed, for both 2D and 3D droplets considering a liquid iron droplet immersed in a very low density gas. Comparisons with the derived analytical solutions are detailed and presented.

\section{The free-oscillating viscous drop problem}

Hereafter we first present the set of equations to describe the oscillation of a free-surface around a (2D) circular and (3D) spherical shape while considering possible interactions between finite viscous and potential forces. The theory is for whatever value of the viscosity, which is an extension of existing theories.

\subsection{Governing equations}

Consider the motion of a Newtonian liquid of density $\rho^{l}$ and viscosity $\mu^{l}$. Let $\Omega^{l}$ be the time-dependent domain occupied by the liquid and $\Gamma$ its interface with a surrounding low density gas phase or, more simply, with vacuum. The liquid is assumed incompressible with constant density $\rho^{l}$, isothermal and no phase transformation is considered. We also neglect all external forces (gravity, magnetic field, forced gas flow, etc) applied to the liquid. In this framework, the flow is governed by the following Navier-Stokes equations:

$$
\left\{\begin{array}{r}
\rho^{l}\left(\frac{\partial \boldsymbol{u}^{l}}{\partial t}+\nabla \boldsymbol{u}^{l} \cdot \boldsymbol{u}^{l}\right)-\nabla \cdot \boldsymbol{\sigma}^{l}=\mathbf{0} \\
\boldsymbol{\nabla} \cdot \boldsymbol{u}^{l}=0
\end{array}\right.
$$

$\boldsymbol{u}^{l}$ is the liquid velocity vector and $\boldsymbol{\sigma}^{l}$ is the liquid stress tensor given by the incompressible Newtonian constitutive law:

$$
\boldsymbol{\sigma}^{l}=2 \mu^{l} \dot{\boldsymbol{\epsilon}}^{l}-p^{l} \boldsymbol{I}
$$

where $\dot{\boldsymbol{\epsilon}}^{l}=\frac{1}{2}\left[\boldsymbol{\nabla} \boldsymbol{u}^{l}+\left(\boldsymbol{\nabla} \boldsymbol{u}^{l}\right)^{T}\right]$ is the strain-rate tensor, $p^{l}$ is the pressure field in the liquid and $\boldsymbol{I}$ is the identity tensor.

At time $t=0 \mathrm{~s}$, we assume that we know the initial shape of the liquid domain and its velocity field such as:

$$
\Omega^{l}(0)=\Omega_{0}^{l}, \quad \boldsymbol{u}^{l}(x, 0)=\boldsymbol{u}_{0}^{l}(x), \quad \nabla \cdot \boldsymbol{u}_{0}^{l}=0
$$

Since no mass exchange occurs through $\Gamma$, the local mass flux leaving the liquid domain $\rho^{l}\left(\boldsymbol{u}^{l} . \boldsymbol{n}-v_{\Gamma}\right)$ is zero, where $\boldsymbol{n}$ is the unit external normal vector on $\Gamma$ and $v_{\Gamma}$ is its normal velocity. This leads to write the kinematic interface condition as:

$$
\boldsymbol{u}^{l} \cdot \boldsymbol{n}=v_{\Gamma}
$$

The second interface condition, named the dynamic condition, arises from the force balance at the interface $\Gamma$ :

$$
\boldsymbol{\sigma}^{l} \cdot \boldsymbol{n}=-\gamma \kappa \boldsymbol{n}-p_{\text {ext }} \boldsymbol{n}
$$

where $\gamma$ is surface tension at the liquid-gas interface, $\kappa$ is the curvature at the liquid-gas interface and $p_{\text {ext }}$ is the pressure in the surrounding gas phase. Further assuming that the liquid is placed in vacuum, $\Gamma$ is a free surface and $p_{\text {ext }}=0$. 


\subsection{Energy balance}

To obtain the energy balance of the drop, we integrate the scalar product of the second equation of (1) with the velocity vector over $\Omega^{l}$ as follows:

$$
\iiint_{\Omega^{l}}\left[\rho^{l}\left(\frac{\partial \boldsymbol{u}^{l}}{\partial t}+\nabla \boldsymbol{u}^{l} \cdot \boldsymbol{u}^{l}\right)-\boldsymbol{\nabla} \cdot \boldsymbol{\sigma}^{l}\right] \cdot \boldsymbol{u}^{l} d V=0
$$

After integrating by part the stress tensor term, we can write:

$$
\iiint_{\Omega^{l}} \frac{1}{2} \rho^{l}\left(\frac{\partial \boldsymbol{u}^{l^{2}}}{\partial t}+\boldsymbol{u}^{l} \cdot \nabla \boldsymbol{u}^{l^{2}}\right) d V-\iint_{\Gamma} \boldsymbol{\sigma}^{l} \cdot \boldsymbol{n} \cdot \boldsymbol{u}^{l} d S+\iiint_{\Omega^{l}} \boldsymbol{\sigma}^{l}: \nabla \boldsymbol{u}^{l} d V=0
$$

The development of this equation (details are given in the appendix) leads to the following equation:

$$
\frac{d}{d t} \underbrace{\left(\iiint_{\Omega^{l}} \frac{1}{2} \rho^{l} \boldsymbol{u}^{l^{2}} d V\right)}_{E_{k i n}^{l}: \text { kinetic energy }}+\frac{d}{d t} \underbrace{\left(\iint_{\Gamma} \gamma d S\right)}_{E_{\text {pot }}^{l}: \text { potential energy }}+\underbrace{\iiint_{\Omega^{l}} 2 \mu^{l} \dot{\boldsymbol{\epsilon}}^{l}: \dot{\boldsymbol{\epsilon}}^{l} d V}_{\dot{W}_{v i s}^{l}: \text { work of viscous force }}=0
$$

This equation expresses the energy balance:

$$
\frac{d}{d t}\left(E_{k i n}^{l}+E_{p o t}^{l}\right)=-\dot{W}_{v i s}^{l}
$$

\section{Analytical solutions in the framework of the linear theory}

\subsection{Drop shape description}

The physics of oscillation refers to the time and space evolution of the surface at the liquid-gas interface $\Gamma$. As shown in figure 1(a), we look for the solution of $R(\theta, \phi, t)$ for the interface position of a perturbed spherical droplet in 3D assuming axisymmetric variations with respect to z-axis (no dependence on $\phi$ ). In $2 \mathrm{D}$, the solution $R(\theta, t)$ is the interface position of a perturbed cross section through an infinite cylinder as shown in figure 1(b). In the latter case, denoting $z$ the longitudinal axis of the cylinder, the analysis is focused on a cross section of infinitely small portion $\delta z$ defining the liquid domain of surface $\Omega^{l}$ and contour $\Gamma$. We consider that the liquid is Newtonian and the flow is incompressible.

The radius of the free surface at each section of the cylinder can be expressed as the sum of a constant part represented by the equilibrium radius $R_{0}$ and a variable part $f(\theta, t)$ which describes the spatial-temporal variations of the free-surface from its equilibrium shape:

$$
R(\theta, t)=R_{0}+f(\theta, t)
$$

As there is no dependency on $\phi$ angle in 3D axisymmetric configuration, formulation (10) is valid also for $3 \mathrm{D}$ case.

In this paper, we work in the context of small-amplitude variations. Therefore, we can write $f(\theta, t)$ as a linear combination of normal modes denoted by the integer $n$ as follows:

$$
\begin{array}{rr}
f(\theta, t)=\sum_{n=0}^{\infty} \alpha_{n}(t) \cos (n \theta) & \text { for } 2 \mathrm{D} \\
f(\theta, t)=\sum_{n=0}^{\infty} \alpha_{n}(t) P_{n}(\cos (\theta)) & \text { for } 3 \mathrm{D}
\end{array}
$$

where 


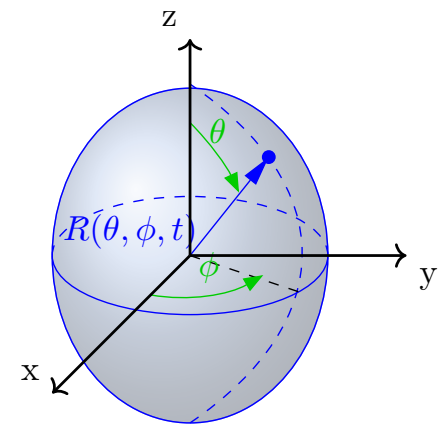

(a) Schematic of 3D axisymetric oscillations occurring in a spherical droplet

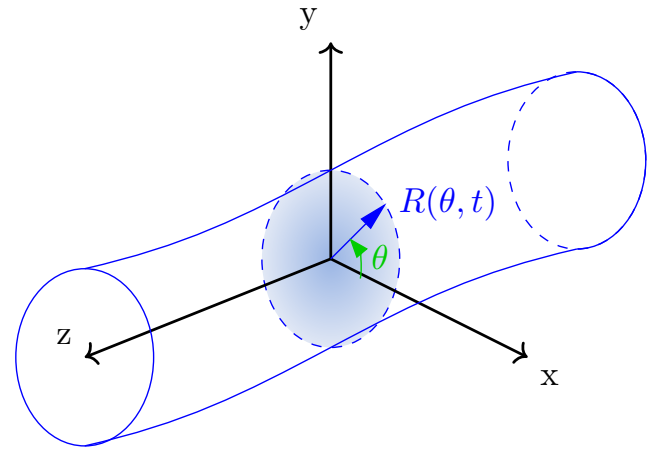

(b) Schematic of 2D oscillations occurring in a cross section of an infinite cylinder

Figure 1: Schematics of the framework of 3D and 2D oscillations

- $\frac{\alpha_{n}(t)}{R_{0}} \ll 1$.

- $P_{n}$ are Legendre polynomials.

- Mode $n=0$ is related to the volume oscillation. We denote by $b_{0}(t)$ the sum $R_{0}+\alpha_{0}(t)$. Thus $b_{0}$ will be determined hereafter by the condition that the volume must remain constant.

- Mode $n=1$ describes the translational oscillations of the mass centre. We consider that the mass centre is fix (zero translational velocity). Consequently $\alpha_{1}(t)=0$.

- Modes $n \geq 2$ describe the shape oscillations around the equilibrium. In the linear theory we can consider that these modes are independent each other and can be treated separately.

Following these considerations, the radius reads:

$$
\begin{array}{rr}
R(\theta, t)=b_{0}(t)+\alpha_{n}(t) \cos (n \theta) & \text { for 2D } \\
R(\theta, t)=b_{0}(t)+\alpha_{n}(t) P_{n}(\cos (\theta)) & \text { for 3D }
\end{array}
$$

Note that in the latter equation and in the following $n \geq 2$.

Assuming that the flow is incompressible, the volume of the oscillating drop is the same as the volume at the equilibrium. Thus:

$$
\begin{array}{r}
V=\delta z \int_{\theta=0}^{2 \pi} \int_{r=0}^{R(\theta, t)} r d r d \theta=\delta z\left(\pi b_{0}^{2}+\frac{1}{2} \pi \alpha_{n}^{2}\right)=\delta z \pi R_{0}^{2} \quad \text { for } 2 \mathrm{D} \\
V=2 \pi \int_{\theta=0}^{\pi} \int_{r=0}^{R(\theta, t)} r^{2} d r \sin (\theta) d \theta=\frac{4 \pi}{3} b_{0}^{3}\left(1+\frac{3}{2 n+1}\left(\frac{\alpha_{n}}{b_{0}}\right)^{2}\right)=\frac{4 \pi}{3} R_{0}^{3} \quad \text { for 3D }
\end{array}
$$

Hence,

$$
\begin{aligned}
& b_{0}=R_{0} \sqrt{1-\frac{1}{2}\left(\frac{\alpha_{n}}{R_{0}}\right)^{2}} \approx R_{0}\left(1-\frac{1}{4}\left(\frac{\alpha_{n}}{R_{0}}\right)^{2}\right) \quad \text { for } 2 \mathrm{D} \\
& b_{0}=R_{0}\left(1+\frac{3}{2 n+1}\left(\frac{\alpha_{n}}{b_{0}}\right)^{2}\right)^{-\frac{1}{3}} \approx R_{0}\left(1-\frac{1}{2 n+1}\left(\frac{\alpha_{n}}{R_{0}}\right)^{2}\right) \quad \text { for } 3 \mathrm{D}
\end{aligned}
$$


Then the radius can be approximated as

$$
\begin{array}{rr}
R(\theta, t)=R_{0}\left(1+\varepsilon_{n} \cos (n \theta)-\frac{1}{4} \varepsilon_{n}^{2}\right) & \text { for } 2 \mathrm{D} \\
R(\theta, t)=R_{0}\left(1+\varepsilon_{n} P_{n}(\cos (\theta))-\frac{1}{2 n+1} \varepsilon_{n}^{2}\right) & \text { for } 3 \mathrm{D}
\end{array}
$$

where $\varepsilon_{n}=\frac{\alpha_{n}}{R_{0}} \ll 1$. It is worth noting that for small-amplitude oscillations the term $\varepsilon_{n}^{2}$ is negligible. However, it will be shown in section 3.3 that potential energy is of the same order as $\varepsilon_{n}^{2}$. Consequently, its presence in equation (15) is crucial for the approximation of the potential energy

The objective now is to find the time-variation of $\varepsilon_{n}$. The outlines of our demonstration are the following: we assume that the flow is irrotational and we find the velocity potential, then we compute the kinetic and potential energies of the drop, next we express the viscous energy dissipation. Finally, we apply the energy balance which leads to a linear differential equation of the quantity $\varepsilon_{n}$.

\subsection{Inner flow modelling}

Following the discussion in [23], the fluid motion can be approximated by an irrotational flow. The velocity field derives from a potential $\boldsymbol{u}^{l}=\nabla \varphi$.

The velocity potential of the $n^{\text {th }}$ mode can be formulated as:

$$
\begin{array}{cc}
\varphi(r, \theta, t)=\beta_{n}(t) r^{n} \cos (n \theta) & \text { for 2D } \\
\varphi(r, \theta, t)=\beta_{n}(t) r^{n} P_{n}(\cos (\theta)) & \text { for 3D }
\end{array}
$$

The coefficient $\beta_{n}$ is obtained from the kinematic boundary condition at the free-surface

$$
u_{r}^{l}(r=R)=\frac{\partial \varphi}{\partial r}(r=R)=\frac{\partial R}{\partial t}
$$

This condition leads to the following approximation which is the same for both $2 \mathrm{D}$ and $3 \mathrm{D}$ analyses: $n \beta_{n} R_{0}^{n-2} \approx \dot{\varepsilon}_{n}$ Hence, we can write the velocity potential as follows:

$$
\begin{array}{r}
\varphi(r, \theta, t)=\frac{1}{n} R_{0}^{2}\left(\frac{r}{R_{0}}\right)^{n} \cos (n \theta) \dot{\varepsilon}_{n}(t) \quad \text { for } 2 \mathrm{D} \\
\varphi(r, \theta, t)=\frac{1}{n} R_{0}^{2}\left(\frac{r}{R_{0}}\right)^{n} P_{n}(\cos (\theta)) \dot{\varepsilon}_{n}(t) \text { for } 3 \mathrm{D}
\end{array}
$$

\subsection{The energy balance}

The kinetic energy of the drop is given by

$$
\begin{array}{r}
E_{k i n}^{l}=\iiint_{V} \frac{1}{2} \rho^{l}\|\nabla \varphi\|^{2} d V=\iint_{S} \frac{1}{2} \rho^{l} \varphi \boldsymbol{\nabla} \varphi \cdot \boldsymbol{n} d S-\iiint_{V} \frac{1}{2} \rho^{l} \varphi \underbrace{\nabla^{2} \varphi}_{=0} d V \\
E_{k i n}^{l}=\frac{1}{2} \rho^{l} \delta z \int_{0}^{2 \pi} \varphi \frac{\partial \varphi}{\partial r} R d \theta \approx \frac{1}{2 n} \pi \rho^{l} R_{0}^{4} \dot{\varepsilon}_{n}^{2} \delta z \quad \text { for } 2 \mathrm{D} \\
E_{k i n}^{l}=\pi \rho^{l} \int_{0}^{\pi} \varphi \frac{\partial \varphi}{\partial r} R^{2} \sin (\theta) d \theta \approx 2 \pi \rho^{l} R_{0}^{5} \frac{1}{n(2 n+1)} \dot{\varepsilon}_{n}^{2} \quad \text { for } 3 \mathrm{D}
\end{array}
$$


Following the work of Rayleigh [7], the potential energy is expressed as:

$$
E_{\text {pot }}^{l}=\gamma\left(S-S_{0}\right)
$$

Where $S$ denotes the area of the free-surface and $S_{0}$ the area of the equilibrium shape.

For the 2D case, the area $S=\delta z P$ where $P$ is the perimeter of the cross section of the cylinder and $S_{0}=2 \pi R_{0}^{2} \delta z$. The perimeter $P$ is calculated through the following integral:

$$
P=\int_{0}^{2 \pi} \sqrt{R^{2}+\left(\frac{\partial R}{\partial \theta}\right)^{2}} d \theta \text { for } 2 \mathrm{D}
$$

Using the expression of the radius $R$ given by the equation (15a) and Taylor expansions we obtain the following approximation

$$
P \approx 2 \pi R_{0}+\frac{1}{2} \pi\left(n^{2}-1\right) R_{0} \varepsilon_{n}^{2}
$$

For the $3 \mathrm{D}$ case, the area $\mathrm{S}$ of the drop is expressed as:

$$
S=2 \pi \int_{0}^{\pi} \sqrt{R^{2}+\left(\frac{\partial R}{\partial \theta}\right)^{2}} R \sin (\theta) d \theta \quad \text { for } 3 \mathrm{D}
$$

After replacing $R$ by the expression (15b), we use Taylor series and some known properties of Legendre polynomials. The area $S$ of the $3 \mathrm{D}$ drop is approximated as follows

$$
S \approx S_{0}+2 \pi R_{0}^{2} \frac{n^{2}+n-2}{2 n+1} \varepsilon_{n}^{2}
$$

where $S_{0}=4 \pi R_{0}^{2}$

Hence,

$$
\begin{array}{rr}
E_{p o t}^{l} \approx \frac{1}{2} \pi\left(n^{2}-1\right) \gamma R_{0} \delta z \varepsilon_{n}^{2} & \text { for } 2 \mathrm{D} \\
E_{p o t}^{l} \approx 2 \pi \gamma R_{0}^{2} \frac{(n+2)(n-1)}{2 n+1} \varepsilon_{n}^{2} & \text { for } 3 \mathrm{D}
\end{array}
$$

Now we can write the rate of change of the total energy:

$$
\begin{array}{rr}
\frac{d E_{t o t}^{l}}{d t}=\pi \frac{1}{n} \rho^{l} R_{0}^{4} \delta z \dot{\varepsilon}_{n}\left[\ddot{\varepsilon}_{n}+n\left(n^{2}-1\right) \frac{\gamma}{\rho^{l} R_{0}^{3}} \varepsilon_{n}\right] & \text { for 2D } \\
\frac{d E_{t o t}^{l}}{d t}=4 \pi \rho^{l} R_{0}^{5} \frac{1}{n(2 n+1)} \dot{\varepsilon}_{n}\left[\ddot{\varepsilon}_{n}+n(n-1)(n+2) \frac{\gamma}{\rho^{l} R_{0}^{3}} \varepsilon_{n}\right] & \text { for 3D }
\end{array}
$$

If the fluid is inviscid $\left(\mu^{l}=0\right)$, the conservation of the total energy $\frac{d E_{t o t}^{l}}{d t}=0$ leads to the solution of a perpetual oscillator of the form $\varepsilon_{n}(t)=A \cos \left(\omega_{n, 0} t+B\right)$ where the angular frequency is

$$
\begin{array}{ll}
\omega_{n, 0}=\sqrt{n(n-1)(n+1) \frac{\gamma}{\rho^{l} R_{0}^{3}}} & \text { for } 2 \mathrm{D} \\
\omega_{n, 0}=\sqrt{n(n-1)(n+2) \frac{\gamma}{\rho^{l} R_{0}^{3}}} & \text { for } 3 \mathrm{D}
\end{array}
$$


as it was found by Rayleigh [7].

As an extension of the work of Rayleigh, we take into consideration, the energy dissipation due to the viscous force of the the bulk liquid. For that, we calculate the rate of the viscous dissipation energy:

$$
\dot{W}_{v i s}^{l}=\iiint_{V} 2 \mu^{l}\left(\dot{\boldsymbol{\epsilon}}^{l}: \dot{\boldsymbol{\epsilon}}^{l}\right) d V
$$

Following the work of Lamb in [8] we can write the volume integral as a surface integral:

$$
\dot{W}_{v i s}^{l}=\iint_{S} \mu^{l} \frac{\partial \boldsymbol{u}^{l^{2}}}{\partial \boldsymbol{n}} d S
$$

The integral over the free surface is calculated as follows:

$$
\begin{array}{rr}
\dot{W}_{v i s}^{l}=\mu^{l} \delta z \int_{0}^{2 \pi} \frac{\partial\|\nabla \varphi\|^{2}}{\partial r} R d \theta & \text { for } 2 \mathrm{D} \\
\dot{W}_{v i s}^{l}=\mu^{l} 2 \pi \int_{0}^{\pi} \frac{\partial\|\boldsymbol{\nabla} \varphi\|^{2}}{\partial r} R^{2} \sin (\theta) d \theta & \text { for } 3 \mathrm{D}
\end{array}
$$

Using Taylor expansions, the dissipation rate can be approximated as

$$
\begin{array}{rr}
\dot{W}_{v i s}^{l} \approx 4 \pi \mu^{l} R_{0}^{2} \delta z(n-1) \dot{\varepsilon}_{n}^{2} & \text { for } 2 \mathrm{D} \\
\dot{W}_{v i s}^{l} \approx 8 \pi \mu^{l} R_{0}^{3} \frac{n-1}{n} \dot{\varepsilon}_{n}^{2} & \text { for 3D }
\end{array}
$$

We can now write the energy balance given in (9). This leads to the following linear second order Ordinary Differential Equation (ODE)

$$
\ddot{\varepsilon}_{n}+2 \lambda_{n} \dot{\varepsilon}_{n}+\omega_{n, 0}^{2} \varepsilon_{n}=0
$$

where

$$
\begin{aligned}
\lambda_{n}=2 n(n-1) \frac{\mu^{l}}{\rho^{l} R_{0}^{2}} & \text { for } 2 \mathrm{D} \\
\lambda_{n}=(2 n+1)(n-1) \frac{\mu^{l}}{\rho^{l} R_{0}^{2}} & \text { for } 3 \mathrm{D}
\end{aligned}
$$

The form of the solution of the ODE (33) depends on the sign of its reduced discriminant

$$
\Delta_{n}^{\prime}=\lambda_{n}^{2}-\omega_{n, 0}^{2}
$$

When $\Delta_{n}^{\prime}>0$ the viscosity is dominant over the surface tension. The solution corresponds to a decay to the equilibrium shape without oscillations. This behaviour is known as the over-damped regime. When $\Delta_{n}^{\prime}<0$ the surface tension is dominant over viscosity. The solution corresponds to oscillations about the equilibrium shape with a decreasing amplitude and a frequency lower than the frequency of an inviscid fluid. In this case we talk about the under-damped regime. In the particular case when $\Delta_{n}^{\prime}=0$, the solution is a rapid relaxation to the equilibrium form without any oscillation. This is known as the critically damped regime.

Without detailing the well-known procedure for solving a linear second order ordinary differential equation, we synthesise, in which follows, the 3 different solutions of the ODE 
- Case $\Delta_{n}^{\prime}>0$ : overdamped regime

$$
\varepsilon_{n}(t)=e^{-\lambda_{n} t}\left[\varepsilon_{n}(0) \cosh \left(\sqrt{\Delta_{n}^{\prime}} t\right)+\frac{\dot{\varepsilon}_{n}(0)+\lambda_{n} \varepsilon_{n}(0)}{\sqrt{\Delta_{n}^{\prime}}} \sinh \left(\sqrt{\Delta_{n}^{\prime}} t\right)\right]
$$

- Case $\Delta_{n}^{\prime}=0$ : Critically damped regime

$$
\varepsilon_{n}(t)=e^{-\lambda_{n} t}\left[\left(\dot{\varepsilon}_{n}(0)+\lambda_{n} \varepsilon_{n}(0)\right) t+\varepsilon_{n}(0)\right]
$$

- Case $\Delta_{n}^{\prime}<0$ : underdamped regime

$$
\varepsilon_{n}(t)=e^{-\lambda_{n} t} \varepsilon_{n, \max } \cos \left(\omega_{n} t+\zeta_{n}\right)
$$

where

$$
\left\{\begin{array}{r}
\omega_{n}=\sqrt{\omega_{n, 0}^{2}-\lambda_{n}^{2}} \\
\varepsilon_{n, \text { max }}^{2}=\varepsilon_{n}^{2}(0)+\left(\frac{\dot{\varepsilon}_{n}(0)+\lambda_{n} \varepsilon_{n}(0)}{\sqrt{-\Delta_{n}^{\prime}}}\right)^{2} \\
\tan \zeta_{n}=-\frac{\dot{\varepsilon}_{n}(0)+\lambda_{n} \varepsilon_{n}(0)}{\varepsilon_{n}(0) \sqrt{-\Delta_{n}^{\prime}}} \\
\cos \left(\zeta_{n}\right) \varepsilon_{n}(0) \geq 0
\end{array}\right.
$$

\section{Numerical framework}

We consider a system composed of two domains: liquid domain denoted $\Omega_{l}$ and gas domain denoted $\Omega_{g}$. The whole domain is $\Omega=\Omega_{l} \cup \Omega_{g}$ and the gas-liquid interface is $\Gamma=\Omega_{l} \cap \Omega_{g}$. Both phases are assumed incompressible, isothermal and no phase transformation is considered. The numerical framework used is based on a full Eulerian finite element approach for solving two-fluid flows. The Level-Set method is used to describe the evolution of the interface. A Variational MultiScale (VMS) stabilized finite element method is proposed to solve the Navier-Stokes equations including both mass conservation and momentum balance equations. The surface tension force is modelled as a volume force by using the Continuum Surface Force (CSF) method.

\subsection{Level-Set method}

The Level-Set (LS) method relies on an implicit representation of the interface via a continuous function whose the zero level corresponds to the interface. The common way to define the LS function is to consider the signed geometrical distance function to the interface defined at any time $t$ and at each position $\boldsymbol{x}$ by

$$
\phi(\boldsymbol{x}, t)=\left\{\begin{array}{rll}
d(\boldsymbol{x}, \Gamma) & \text { if } & \boldsymbol{x} \in \Omega_{l} \\
0 & \text { if } & \boldsymbol{x} \in \Gamma \\
-d(\boldsymbol{x}, \Gamma) & \text { if } & \boldsymbol{x} \in \Omega_{g}
\end{array}\right.
$$

where $d(\boldsymbol{x}, \Gamma)$ is the distance from the position $\boldsymbol{x}$ to the interface $\Gamma$. Here the positive values are arbitrarily chosen in the liquid domain. The time-evolution of the interface is given by the following transport equation:

$$
\frac{\partial \phi}{\partial t}+\boldsymbol{u} \cdot \boldsymbol{\nabla} \phi=0
$$

where $\boldsymbol{u}$ denotes the flow velocity vector. The zero isovalue resulting from equation (41) represents the position of the interface whereas the non-zero levels are not guaranteed to represent the geometrical distance 
to the interface, especially when the velocity field $\boldsymbol{u}$ is not uniform. Therefore, a re-initialization of the LS function is needed in order to recover the intrinsic property of the distance function which is $\|\nabla \phi\|=1$. Solving the Hamilton-Jacobi equation (42) is a classical way for re-distancing.

$$
\frac{\partial \phi}{\partial \tau}+s(\phi)(\|\nabla \phi\|-1)=0
$$

where $\tau$ is a pseudo-time and $s(\phi)$ is the sign of the Level-Set function $\phi$. This re-initialization method requires solving a number of sub-iterations at each time step, which can be time-consuming. To overcome the need of two different solvers for transport and re-initialization, the coupled convection-re-initialization equation (43) combines both equations (41) and (42).

$$
\frac{\partial \phi}{\partial t}+\boldsymbol{U} \cdot \nabla \phi=k s(\phi)
$$

where $k$ is a numerical constant dimensionally homogeneous to a velocity and

$$
\boldsymbol{U}=\boldsymbol{u}+k s(\phi) \frac{\nabla \phi}{\|\nabla \phi\|}
$$

It should be noted that this auto-reinitialization transport equation (43) is solved in finite-element by means of Streamline Upwind PetrovGalerkin (SUPG) method known to mitigate the numerical instabilities. See [24] for more numerical details.

\subsection{Mass correction method}

Despite its mathematical simplicity and attractive ability to handle complex geometries, the classical Level-Set method (distance function) suffers from mass conservation issues. In fact, after the transport of LS function, the volume (consequently the mass, for an incompressible flow) of the object described by the LS $\left(\Omega_{l}\right.$ or $\left.\Omega_{g}\right)$ can be lost or gained due to numerical errors within each time step. After several time-steps the accumulation of numerical errors can yield a significant gain/loss of mass. In the present work we use a simple correction method to enforce the volume (thus the mass) to be invariant. The principle of this correction method is to shift the zero-isovalue of the LS function (distance function) by some signed distance $c_{\phi}$

$$
\phi^{\operatorname{corr}}(x, t)=\phi(x, t)+c_{\phi}(t)
$$

where $\phi^{\text {corr }}$ is the corrected LS function and $\phi$ is the transported LS resulting from (43). The signed distance $c_{\phi}$ is computed as the volume difference, taking as a reference the initial volume, over the area of the interface $A_{\Gamma}$

$$
c_{\phi}(t)=\frac{V(t)-V(0)}{A_{\Gamma}(t)}
$$

It should be mentioned that this adjustment $\left|c_{\phi}\right|$ should be small enough (not greater than $O\left(h^{2}\right)$ for $\mathbb{P} 1$ elements where $h$ is the mesh size according to [25]) to preserve the shape of the interface. This means that the numerical disretization errors within each time step must be minimised as possible. For this purpose, we use a high order time-discretization scheme $\left(2^{\text {nd }}\right.$ order is sufficient) for solving the transport equation $(43)$.

\subsection{Mixing law}

A monolithic resolution type is adopted. This enables the resolution of a single set of equations for both fluids in the whole domain. The discontinuity of properties at the interface raises computational challenges. For this reason, switching from one fluid to the other is operated continuously by a smoothed Heaviside 
function and an arithmetic mixing law. This requires considering a transition zone centred at the interface with a half-thickness $e$. The Heaviside function which indicates the presence of the liquid is then defined as

$$
H(\phi)= \begin{cases}1 & \text { if } \phi>e \\ 0 & \text { if } \phi<-e \\ \frac{1}{2}\left[1+\frac{\phi}{e}+\frac{1}{\pi} \sin \left(\frac{\pi \phi}{e}\right)\right] & \text { if } \quad|\phi| \leq e\end{cases}
$$

where the thickness $e$ is taken as 2 to 3 times the mesh size. Let $\psi$ be a fluid property such as density, viscosity, etc. $\psi$ is indexed with $\mathrm{l}$ when it is a liquid property and with $\mathrm{g}$ when it is a gas property. We express the mixed property $\langle\psi\rangle$ defined in the whole domain by an arithmetic law as

$$
<\psi>=\psi_{l} H+\psi_{g}(1-H)
$$

\subsection{Surface tension force}

Surface tension is introduced using the Continuum Surface Force (CSF) method [26]. This approach consists in including the surface tension as a volume source term in Navier-Stokes equations via a smoothed Dirac function $\delta_{e}(\phi)$ centred at the interface. Accordingly, the surface tension force $f_{S T}$ is expressed as follows:

$$
\boldsymbol{f}_{\boldsymbol{S T}}=-\gamma \kappa \delta_{e}(\phi) \boldsymbol{n}
$$

where $\kappa$ is the mean curvature and $n$ is the normal to the surface $\Gamma$. These geometrical properties of the interface are directly computed thanks to the Level-Set method using the following expressions:

$$
\begin{gathered}
\boldsymbol{n}=\frac{\boldsymbol{\nabla} \phi}{\|\boldsymbol{\nabla} \phi\|} \\
\kappa=-\boldsymbol{\nabla} \cdot \boldsymbol{n}
\end{gathered}
$$

Following these considerations, the surface tension force is calculated from the Level-Set function as:

$$
\boldsymbol{f}_{\boldsymbol{S T}}=\gamma \delta_{e}(\phi) \boldsymbol{\nabla} \cdot\left(\frac{\boldsymbol{\nabla} \phi}{\|\boldsymbol{\nabla} \phi\|}\right) \frac{\boldsymbol{\nabla} \phi}{\|\boldsymbol{\nabla} \phi\|}
$$

\subsection{Monolithic formulation of Navier-Stokes equations}

In this section, we present the Variational Multiscale (VMS) method to solve the unsteady two-phase Navier-Stokes equations. To fix a notation, let $\Omega \subset \mathbb{R}^{d}$ be the fluid domain, where $d$ is the space dimension, and $\partial \Omega$ its boundary. The strong form of the incompressible Navier Stokes equations reads:

$$
\left\{\begin{array}{l}
\rho\left(\frac{\partial \boldsymbol{u}}{\partial t}+\boldsymbol{u} \cdot \boldsymbol{\nabla} \boldsymbol{u}\right)-\boldsymbol{\nabla} \cdot \boldsymbol{\sigma}=\boldsymbol{f}_{\boldsymbol{S T}} \\
\boldsymbol{\nabla} \cdot \boldsymbol{u}=0
\end{array}\right.
$$

where $t \in[0, T]$ is the time, $\boldsymbol{u}(\boldsymbol{x}, t)$ the velocity, $p(\boldsymbol{x}, t)$ the pressure, $\rho$ the density and $\boldsymbol{f}_{\boldsymbol{S T}}$ is the surface tension force. The Cauchy stress tensor for a Newtonian fluid is given by:

$$
\boldsymbol{\sigma}=2 \mu \boldsymbol{\epsilon}(\boldsymbol{u})-p \boldsymbol{I}_{d}
$$

with $\boldsymbol{I}_{d}$ the $d$-dimensional identity tensor and $\mu$ the dynamic viscosity. In order to close the problem, Equations (53) are subjected to boundary and initial conditions to be specified.

The weak form of problem (53) can be obtained by multiplication with test functions and integration by parts. Let $H^{1}(\Omega)$ be the Sobolev space of square integrable functions whose distributional derivatives are 
square integrable, and let $V \subset\left[H^{1}(\Omega)\right]^{d}$ be a functional space properly chosen according to the boundary conditions. Finally, let $Q=\left\{q \in L^{2}(\Omega): \int_{\Omega} q=0\right\}$. If we denote by $(\cdot, \cdot)$ the $L^{2}$ inner product over the computational domain $\Omega$, the weak form of problem (53) reads, under the assumption of homogeneous Dirichlet boundary conditions:

$$
\left\{\begin{array}{l}
\text { Find }(\boldsymbol{u}, p) \in V \times Q \text { such that: } \\
\rho\left[\left(\partial_{t} \boldsymbol{u}, \boldsymbol{w}\right)+(\boldsymbol{u} \cdot \nabla \boldsymbol{u}, \boldsymbol{w})\right]+(2 \mu \boldsymbol{\epsilon}(\boldsymbol{u}): \boldsymbol{\epsilon}(\boldsymbol{w}))-(p, \nabla \cdot \boldsymbol{w})=(\boldsymbol{f}, \boldsymbol{w}), \quad \forall \boldsymbol{w} \in V \\
(\nabla \cdot \boldsymbol{u}, q)=0, \quad \forall q \in Q
\end{array}\right.
$$

Based on a mesh $\mathcal{K}_{h}$ of $\Omega$ made of $N_{e l}$ elements $K$, the functional spaces for the velocity $V$ and for the pressure $Q$ are approximated by the finite dimensional spaces $V_{h}$ and $Q_{h}$ respectively. It is well known that the stability of the semi-discrete formulation requires an appropriate choice of the finite element spaces $V_{h}$ and $Q_{h}$, that must to fulfill the Babuska-Brezzi condition [27]. Accordingly, the standard Galerkin method with the P1/P1 element (i.e. the same piecewise linear space for $V_{h}$ and $Q_{h}$ ) is not stable. Moreover, convectiondominant problems also lead to a loss of coercivity in formulation (55), hence numerical oscillations that end up polluting the whole solution. In this work, we use a Variational MultiScale method $[28,29]$ meant to circumvent both problems. The basic idea is to split all unknowns into two components, a coarse one and a fine one, that correspond to different scales or levels of resolution. In practice, we solve the fine scales in an approximate manner and then replace their effect into the large-scale equation. We present here only an outline of the method, and the reader is referred to [30] for extensive details about the formulation.

Let us split the velocity and the pressure fields into resolvable coarse-scale and unresolved fine-scale components, i.e., $\boldsymbol{u}=\boldsymbol{u}_{h}+\boldsymbol{u}^{\prime}$ and $p=p_{h}+p^{\prime}$, where the subscript $h$ is used hereafter to denote the finite element (coarse) component, and the prime is used for the so called subgrid scale (fine) component. The same decomposition can be applied to the weighting functions, hence $\boldsymbol{w}=\boldsymbol{w}_{h}+\boldsymbol{w}^{\prime}$ and $q=q_{h}+q^{\prime}$. The enriched functional spaces are defined as $V=V_{h} \oplus V^{\prime}, V_{0}=V_{h, 0} \oplus V_{0}^{\prime}$ and $Q=Q_{h} \oplus Q^{\prime}$. The discretized, finite element approximation for the time-dependent Navier-Stokes problem therefore reads

$$
\begin{aligned}
& \text { Find }(\boldsymbol{u}, p) \in V \times Q \text { such that: } \\
& \left\{\begin{aligned}
& \rho\left(\partial_{t}\left(\boldsymbol{u}_{h}+\boldsymbol{u}^{\prime}\right)\right.\left.,\left(\boldsymbol{w}_{h}+\boldsymbol{w}^{\prime}\right)\right)+\rho\left(\left(\boldsymbol{u}_{h}+\boldsymbol{u}^{\prime}\right) \cdot \nabla\left(\boldsymbol{u}_{h}+\boldsymbol{u}^{\prime}\right),\left(\boldsymbol{w}_{h}+\boldsymbol{w}^{\prime}\right)\right) \\
&+\left(2 \mu \boldsymbol{\epsilon}\left(\boldsymbol{u}_{h}+\boldsymbol{u}^{\prime}\right): \boldsymbol{\epsilon}\left(\boldsymbol{w}_{h}+\boldsymbol{w}^{\prime}\right)\right) \\
&-\left(\left(p_{h}+p^{\prime}\right), \nabla \cdot\left(\boldsymbol{w}_{h}+\boldsymbol{w}^{\prime}\right)\right)=\left(\mathbf{f},\left(\boldsymbol{w}_{h}+\boldsymbol{w}^{\prime}\right)\right), \quad \forall \boldsymbol{w} \in V_{0} \\
&\left(\nabla \cdot\left(\boldsymbol{u}_{h}+\boldsymbol{u}^{\prime}\right),\left(q_{h}+q^{\prime}\right)\right)_{\Omega}=0, \quad \forall q \in Q .
\end{aligned}\right.
\end{aligned}
$$

In order to derive the stabilized formulation, we split Equation (56) into a large-scale and a fine-scale problem. The fine-scale problem is defined on element interiors. Under several assumptions regarding the time-dependency and the non-linearity of the momentum equation of the subscale system detailed in [30], the fine-scale solutions $\boldsymbol{u}^{\prime}$ and $p^{\prime}$ are written in terms of the time-dependent large-scale variables using consistently derived residual-based terms. Consequently, we can use static condensation, that consists in substituting directly $\boldsymbol{u}^{\prime}$ and $p^{\prime}$ into the large-scale problem, which gives rise to additional terms in the Finite Element formulation, tuned by a local stabilizing parameter. These terms are responsible for the enhanced stability compared to the standard Galerkin formulation. The large-scale system finally reads:

$$
\left\{\begin{array}{c}
\rho\left(\partial_{t} \boldsymbol{u}_{h}, \boldsymbol{w}_{h}\right)_{\Omega}+\left(\rho \boldsymbol{u}_{h} \cdot \nabla \boldsymbol{u}_{h}, \boldsymbol{w}_{h}\right)_{\Omega} \\
-\sum_{K \in \mathcal{T}_{h}}\left(\tau_{1} \boldsymbol{\mathcal { R }}_{\mathrm{M}}, \rho \boldsymbol{u}_{h} \nabla \boldsymbol{w}_{h}\right)_{K}+\left(2 \mu \boldsymbol{\epsilon}\left(\boldsymbol{u}_{h}\right): \boldsymbol{\epsilon}\left(\boldsymbol{w}_{h}\right)\right)_{\Omega} \\
-\left(p_{h}, \nabla \cdot \boldsymbol{w}_{h}\right)_{\Omega}-\sum_{K \in \mathcal{T}_{h}}\left(\tau_{2} \mathcal{R}_{\mathrm{C}}, \nabla \cdot \boldsymbol{w}_{h}\right)_{K}=\left(\mathbf{f}, \boldsymbol{w}_{h}\right)_{\Omega}, \quad \forall \boldsymbol{w}_{h} \in V_{h, 0} \\
\left(\nabla \cdot \boldsymbol{u}_{h}, q_{h}\right)_{\Omega}-\sum_{K \in \mathcal{T}_{h}}\left(\tau_{1} \boldsymbol{\mathcal { R }}_{\mathrm{M}}, \nabla q_{h}\right)_{K}=0, \quad \forall q_{h} \in Q_{h}
\end{array}\right.
$$

where $(\cdot, \cdot)_{\Omega}$ represents the inner product over the whole domain $\Omega,(\cdot, \cdot)_{K}$ is the inner product over Element $K, \boldsymbol{\mathcal { R }}_{\mathrm{M}}$ and $\mathcal{R}_{\mathrm{C}}$ are momentum and continuity residual expressed as

$$
\begin{aligned}
& \mathcal{R}_{\mathrm{M}}=\mathbf{f}-\rho \partial_{t} \boldsymbol{u}_{h}-\rho \boldsymbol{u}_{h} \cdot \nabla \boldsymbol{u}_{h}-\nabla p_{h} \\
& \mathcal{R}_{\mathrm{C}}=-\nabla \cdot \boldsymbol{u}_{h}
\end{aligned}
$$


and $\tau_{1}$ and $\tau_{2}$ are stabilization parameters for which we adopt the definition proposed in [31]:

$$
\begin{aligned}
& \tau_{1}=\left[\left(\frac{2 \rho\left\|\boldsymbol{u}_{h}\right\|_{K}}{h_{K}}\right)^{2}+\left(\frac{4 \mu}{h_{K}^{2}}\right)^{2}\right]^{-\frac{1}{2}}, \\
& \tau_{2}=\left[\left(\frac{\mu}{\rho}\right)^{2}+\left(\frac{c_{2}\left\|\boldsymbol{u}_{h}\right\|_{K}}{c_{1} h_{K}}\right)^{2}\right]^{\frac{1}{2}}
\end{aligned}
$$

where $h_{K}$ is the characteristic length of the element and $c_{1}$ and $c_{2}$ are algorithmic constants. We take them as $c_{1}=4$ and $c_{2}=2$ for linear elements [31]. Compared to the standard Galerkin method, the proposed stabilized formulation involves additional integrals that are evaluated element-wise. These additional terms represent the stabilizing effect of the sub-grid scales and are introduced in a consistent way in the Galerkin formulation. They allow avoiding instabilities caused by both dominant convection terms and incompatible approximation spaces.

\section{Results and discussion}

\section{1. $2 D$ simulation of single oscillation modes}

In this section the results of simulations performed with the numerical framework presented in section 2 , are compared with the results of the analytical solution developed in section 3 for the $2 \mathrm{D}$ case. We consider a liquid iron droplet suspended in the air in the absence of gravity. The area of the droplet is $\pi R_{0}^{2}$ where $R_{0}=3 \times 10^{-3} \mathrm{~m}$ is the radius of the equilibrium shape of the droplet (dashed white line in figure 2). The properties of the materials are taken at the temperature of $1800 \mathrm{~K}$. The density of the liquid iron is $\rho^{l}=7040 \mathrm{~kg} \cdot \mathrm{m}^{-3}$ whereas the air density is $\rho^{g}=0.19 \mathrm{~kg} \cdot \mathrm{m}^{-3}$. The dynamic viscosities are $\mu^{l}=5.8510^{-3}$ Pa.s and $\mu^{g}=5.8210^{-5}$ Pa.s for the liquid iron and air respectively. The ratios of density and viscosity are great enough to neglect the effects of air on the dynamics of the droplet. This condition is necessary in order to be in accordance with the previous theoretical analysis which supposes that the droplet is force-free. The whole system is enclosed in a cavity of side size $8 \times R_{0}$ (see figure 2). The initial shape of the droplet is slightly deformed from the circular equilibrium shape. The initial shape of each mode is described by

$$
R(\theta, t=0)=R_{0}\left(1+\varepsilon_{n, 0} \cos (n \theta)-\frac{1}{4} \varepsilon_{n, 0}^{2}\right)
$$

where $\varepsilon_{n, 0}=0.02$.

Different modes of deformation ( $n$ from 2 to 7 ) are investigated. The droplet is released from a static state which means that $\boldsymbol{u}(r, \theta, t=0)=\mathbf{0}$. The computational domain is discretized into unstructured mesh of triangles. The mesh is refined inside a circular region which covers the liquid domain (see Figure 2) with a mesh characteristic size $h=10^{-5} \mathrm{~m}$. It consists of 50848 elements and 25437 nodes. 


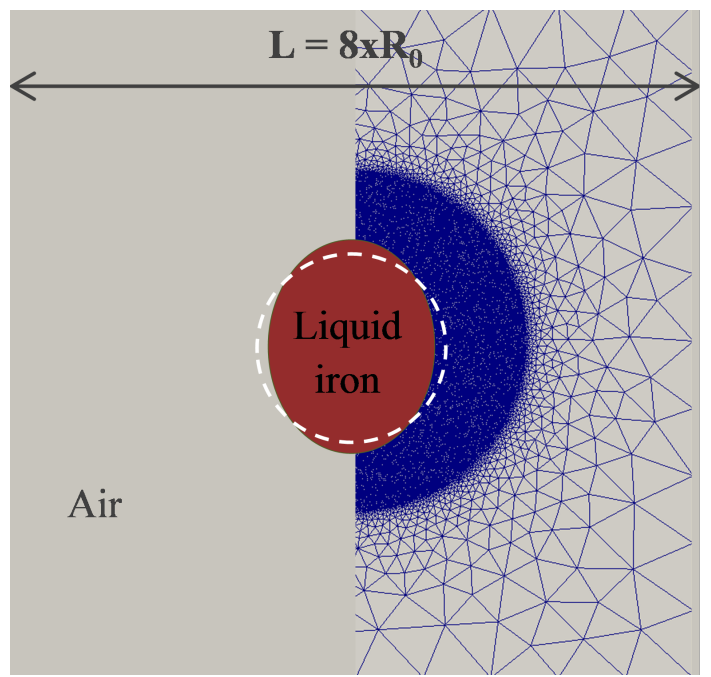

Figure 2: 2D simulation setup

Following the previous theoretical analysis of the droplet dynamics, the behaviour of the free-surface depends on the balance of forces between surface tension and viscosity. This balance of forces can be determined from the sign of the reduced discriminant $\Delta_{n}^{\prime}=\lambda_{n}^{2}-\omega_{n, 0}^{2}$ which has the same $\operatorname{sign}$ as $\frac{4 n(n-1)}{n+1}-$ $\frac{\rho^{l} R_{0} \gamma}{\mu^{l^{2}}}$.

Here $\frac{\rho^{l} R_{0} \gamma}{\mu^{l^{2}}}$ is of the order of $10^{6}$. For the range of modes considered here it is obvious that $\frac{4 n(n-1)}{n+1}$ is much lower than $\frac{\rho^{l} R_{0} \gamma}{\mu^{l^{2}}}$. So $\Delta_{n}^{\prime}<0$. It means that surface tension is dominant over viscosity, thus, the behaviour of the iron droplet, in the current range of modes, corresponds to the under-damped regime. This qualitative analysis of balance of forces is confirmed by the following table which gives the values of $\lambda_{n}^{2}, \omega_{n, 0}^{2}$ and $\Delta_{n}^{\prime}$ for each mode.

\begin{tabular}{lcccccc} 
Mode & 2 & 3 & 4 & 5 & 6 & 7 \\
\hline$\lambda_{n}^{2}$ & 0.14 & 1.23 & 4.91 & 13.64 & 30.69 & 60.15 \\
\hline$\omega_{n, 0}^{2}$ & $7.8710^{4}$ & $2.9510^{5}$ & $7.0810^{5}$ & $1.3710^{6}$ & $2.3610^{6}$ & $3.7110^{6}$ \\
\hline$\Delta_{n}^{\prime}$ & $-7.8710^{4}$ & $-2.9510^{5}$ & $-7.0810^{5}$ & $-1.3710^{6}$ & $-2.3610^{6}$ & $-3.7110^{6}$
\end{tabular}

Table 1: The values of $\lambda_{n}^{2}, \omega_{n, 0}^{2}$ and $\Delta_{n}^{\prime}$ for each oscillation mode

The initial conditions leads to $\dot{\varepsilon}_{n}(0)=0$ and $\varepsilon_{n}(0)=\varepsilon_{n, 0}$ Following the values of the table 5.1, the observed oscillation frequency $\sqrt{\Delta_{n}^{\prime}}$, in this case, are approximately equal to the frequency of a non-viscous liquid $\omega_{n, 0}$. The solution given by the equation (38) can be approximated as:

$$
\varepsilon_{n}(t) \approx \varepsilon_{n, 0} e^{-\lambda_{n} t} \cos \left(\omega_{n, 0} t\right)
$$

Figure 3 presents the time-evolution of the liquid domain (red region) depicted by positive-Level-Set function $(\phi>=0)$ for different modes. The black arrows represent the inner velocity field. We can clearly highlight the robustness of the proposed numerical framework to handle different shapes and their oscillations.

To assess the accuracy of these simulations, we extract from the simulation of each mode the time-evolution of the droplet radius in $\theta=0$ direction. Then, we compare the extracted temporal signals to the derived 
analytical solution given by equations (63), (28a), (34a) and (15a). The numerical signals superimposed to the theoretical ones are shown in figure 4. A very good agreement is obtained for different modes, which confirms again the accuracy and the robustness of the proposed numerical framework.

The numerical oscillation frequency of each mode $n$ is extracted from the frequency spectrum of the radius by means of the Fast Fourier Transform (FFT) whereas the damping coefficient is obtained by fitting the envelop of the temporal signal to $e^{-\lambda_{n} t}$ using the least squares algorithm. Figure 5 reports the variation of the oscillation frequency and the damping rate with respect to the oscillation mode $n$. The numerical results are again in a good quantitative agreement with the analytical formulae (34a) and (28a). 

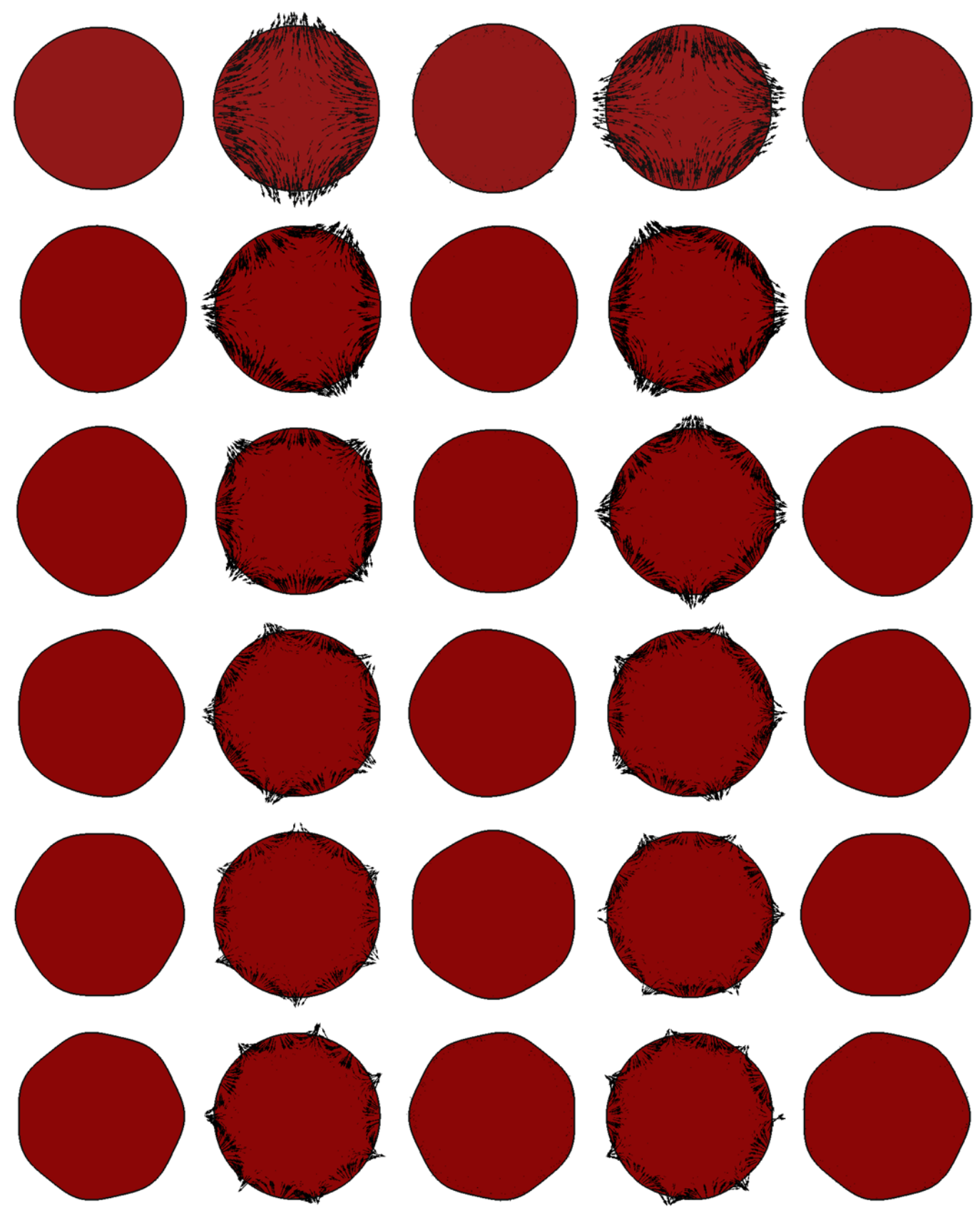

Figure 3: The time-evolution of the droplet shape over the first oscillation period for modes from $n=2$ (upper row) to $n=7$ (lower row). From the left column to the right, time corresponds to $t=0, \frac{T_{n}}{4}, \frac{T_{n}}{2}, \frac{3 T_{n}}{4}$ and $T_{n}$ respectively 


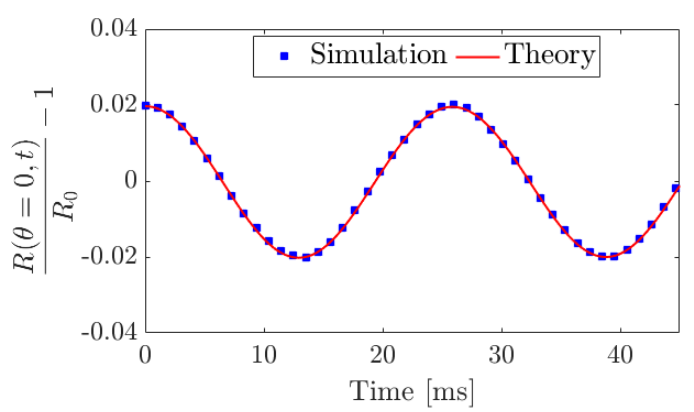

(a) Mode 2

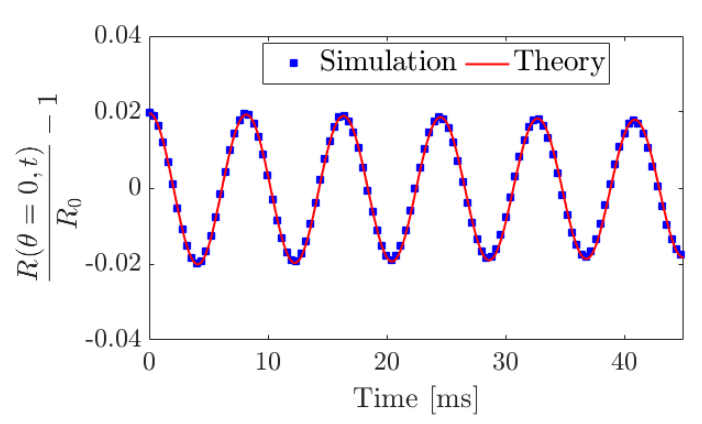

(c) Mode 4

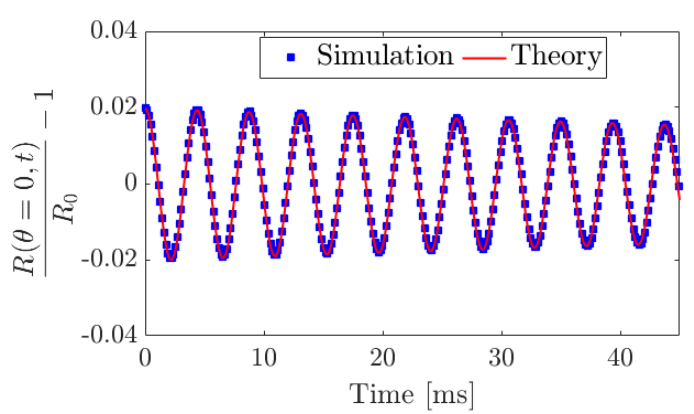

(e) Mode 6

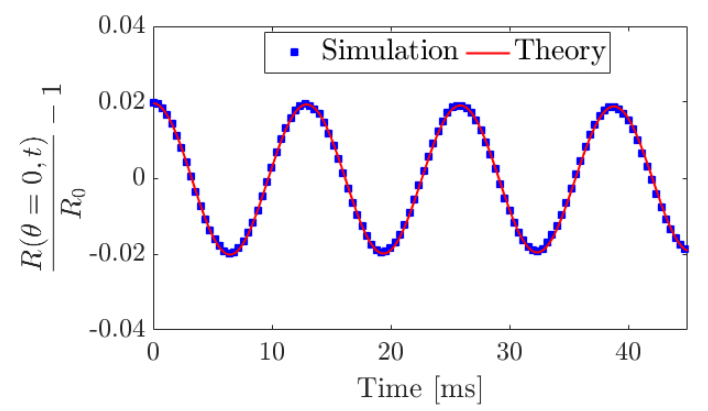

(b) Mode 3

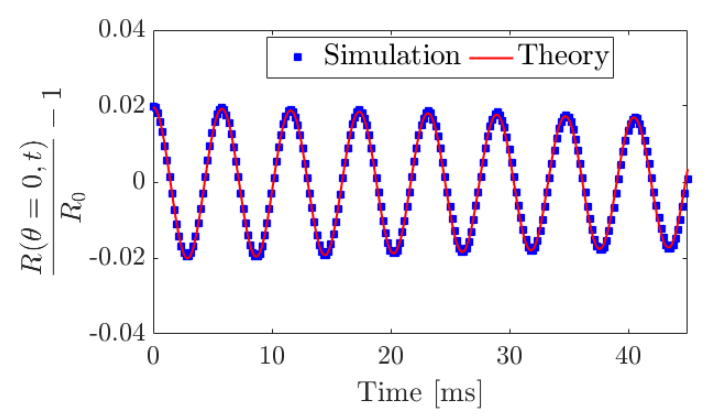

(d) Mode 5

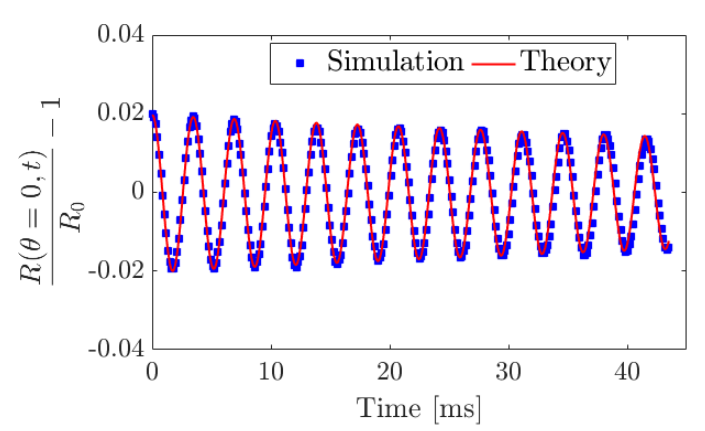

(f) Mode 7

Figure 4: The temporal signals of the droplet's radius in direction $\theta=0$ for modes $n \in \llbracket 2,7 \rrbracket$ 


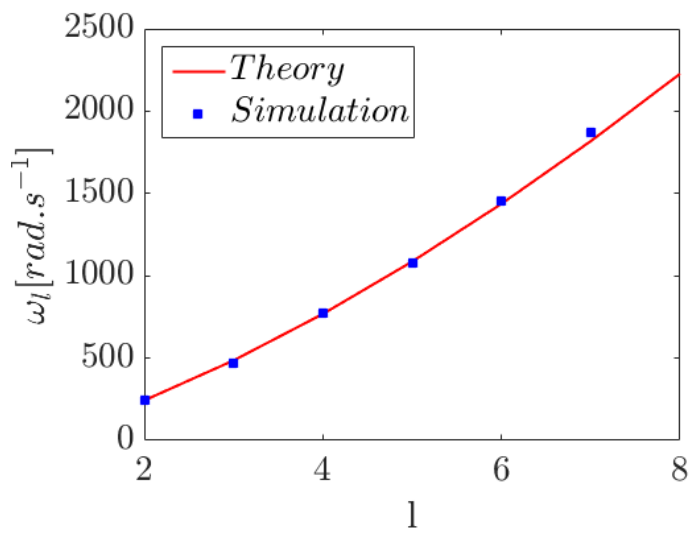

(a) Oscillation frequency

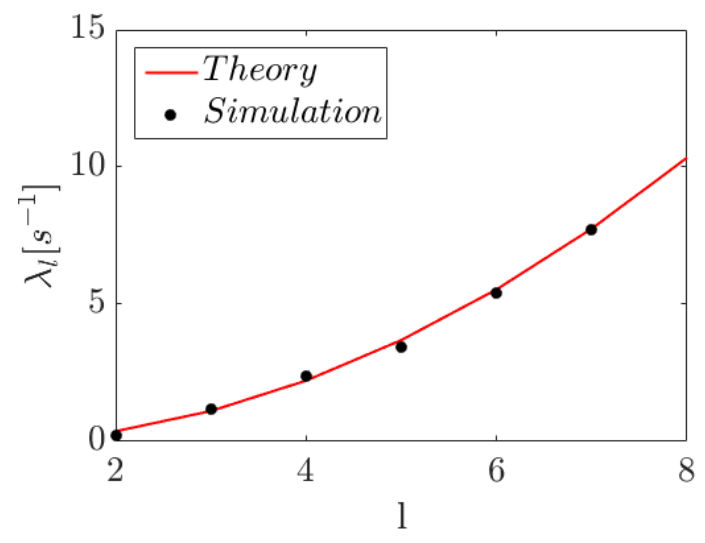

(b) Damping rate

Figure 5: The variation of the oscillation frequency and the damping rate with respect to the oscillation mode

\subsection{D simulation of an arbitrary initial form}

In the previous section, the droplet is initially released from a deformed shape describing a specific single mode. Single mode excitation could be constraining to be set up experimentally. What if the initial shape of the droplet is arbitrary? In this section we try to investigate this question by proposing an initial shape described by equation (64). It describes the combination of several modes. Each mode is rotated from the position given by equation (62) with angle $\psi_{n}$. Here, we fix the highest order mode to 7 and we generate randomly the values of $\varepsilon_{n, 0}$ between $1 \%$ and $2 \%$ (small amplitudes condition) and $\psi_{n}$ between 0 and $2 \pi$. These values are reported in table 5.2.

$$
R(\theta, t=0)=R_{0}\left[1+\sum_{n=2}^{N}\left(\varepsilon_{n, 0} \cos \left(n \theta+\psi_{n}\right)-\frac{1}{4} \varepsilon_{n, 0}^{2}\right)\right]
$$

\begin{tabular}{l|cccccc} 
Mode & 2 & 3 & 4 & 5 & 6 & 7 \\
\hline$\varepsilon_{n, 0}$ & $1.815 \%$ & $1.906 \%$ & $1.127 \%$ & $1.913 \%$ & $1.632 \%$ & $1.098 \%$ \\
\hline$\psi_{n}[\mathrm{rad}]$ & 1.750 & 3.436 & 6.016 & 6.063 & 0.990 & 6.098
\end{tabular}

Table 2: Random values of $\varepsilon_{n, 0}$ and $\psi_{n}$ for each normal mode

In this simulation, material properties are the same as in the previous section. Figure 6 displays the velocity field as well as the shape of the interface at different times. 


$$
\mathrm{t}=0.0 \mathrm{~ms}
$$

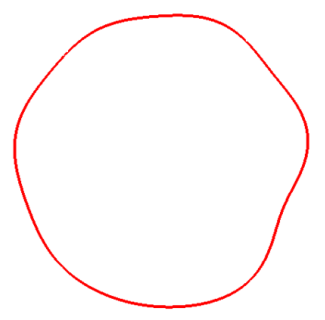

Velocity $[\mathrm{m} / \mathrm{s}]$

$$
2.10 \mathrm{e}-01
$$

1.40e-01

7.00e-02

$0.00 \mathrm{e}+00$ $\mathrm{t}=2.0 \mathrm{~ms}$

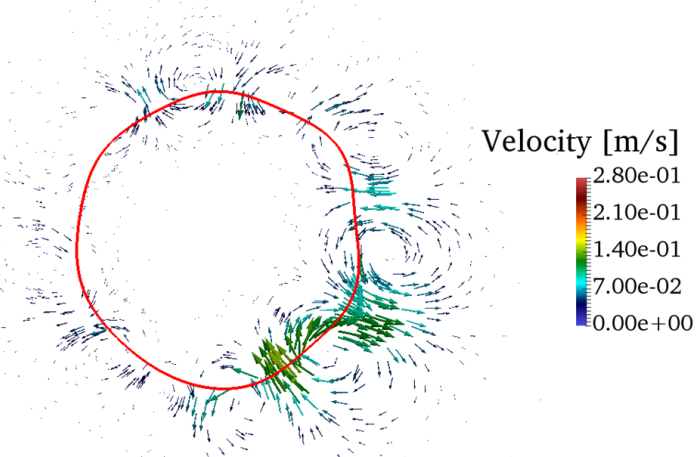

$\mathrm{t}=6.0 \mathrm{~ms}$

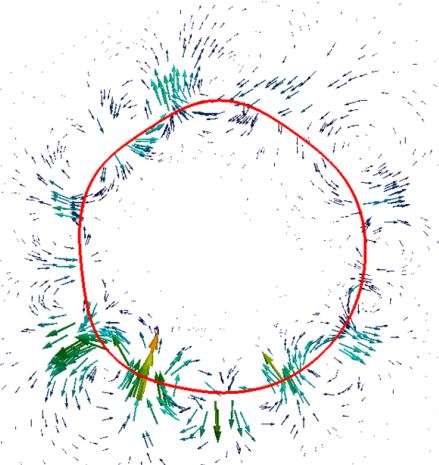

Velocity $[\mathrm{m} / \mathrm{s}]$

2.80e- 01

$1.40 \mathrm{e}-01$

7.00e- 02

$0.00 e+00$

\section{$\mathrm{t}=8.0 \mathrm{~ms}$}

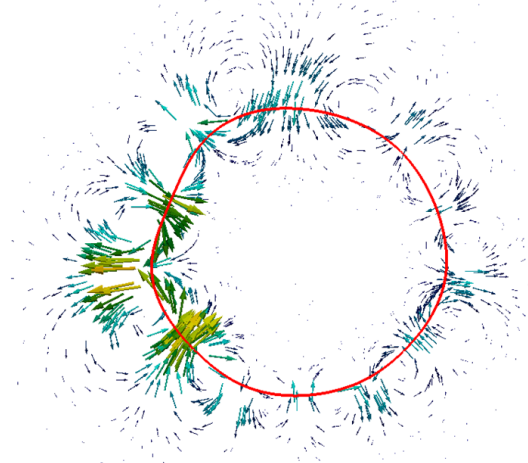

Velocity $[\mathrm{m} / \mathrm{s}]$

2.10e-01

$1.40 \mathrm{e}-01$

7.00e-02
Velocity $[\mathrm{m} / \mathrm{s}]$

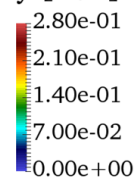

$$
\mathrm{t}=10.0 \mathrm{~ms}
$$

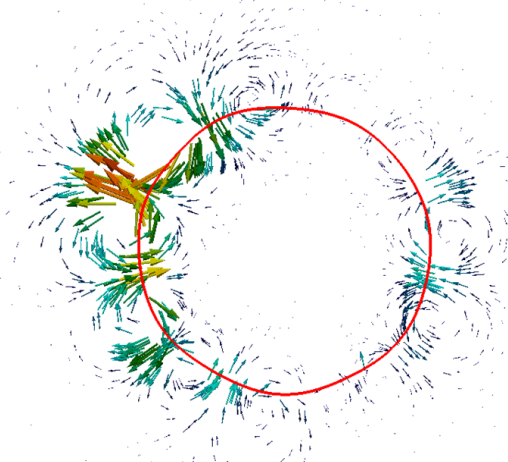

Velocity [m/s]

$2.10 \mathrm{e}-01$

$1.40 \mathrm{e}-01$

$7.00 \mathrm{e}-02$

$0.00 \mathrm{e}+00$

Figure 6: Snapshots of the droplet shape and velocity field at various times

We can show easily from the theoretical analysis detailed in section 3 that the analytical solution of this problem at each time $t$ is expressed as:

$$
R(\theta, t)=R_{0}\left[1+\sum_{n=2}^{7}\left(\varepsilon_{n} \cos \left(n \theta+\psi_{n}\right)-\frac{1}{4} \varepsilon_{n}^{2}\right)\right]
$$


where

$$
\varepsilon_{n}(t) \approx \varepsilon_{n, 0} e^{-\lambda_{n} t} \cos \left(\omega_{n, 0} t+\psi_{n}\right)
$$

The blue line in figure 5.2 corresponds to the simulated temporal variation of the radius of the interface in the direction $\theta=0$. The comparison with the theoretical solution (the red dashed line) shows again a good agreement. We compute the frequency spectrum of the numerical temporal signal via the FFT. It is clear from figure 7 that the spectrum (blue line) displays only the peaks of the modes from 2 to 7 whose the frequencies are represented by the red dashed vertical lines.

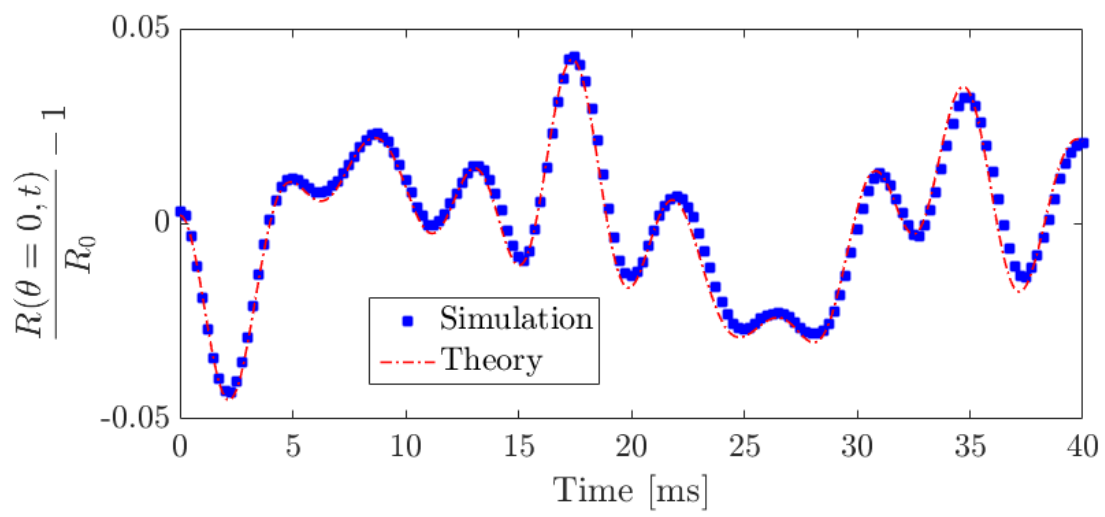

(a) Time evolution of the radius $R(\theta, t)$

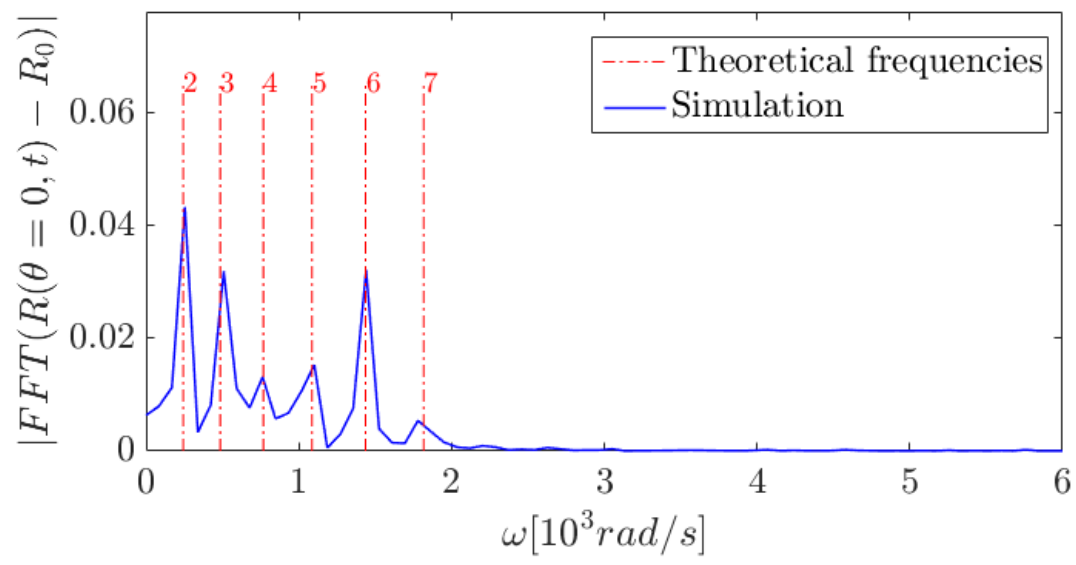

(b) The frequency spectrum of the signal $R(\theta, t)$ given by the FFT

Figure 7: Comparison of simulation results and the analytical solution

The results of this investigation show that if an arbitrary initial shape, slightly deformed from the equilibrium shape, can be written as a combination of normal modes, the linear theory derived in this work can provide the analytical expression of the time-evolution of the radius of the droplet.

\subsection{D simulation of the oscillation of a liquid iron drop}

In this section we perform 3D simulation of the oscillation of a liquid iron drop. The liquid iron droplet is suspended in the air in the absence of gravity and enclosed in a cavity of side size $6 \times R_{0}$. The values of densities and viscosities are the same as in the $2 \mathrm{D}$ case as well as surface tension value. The droplet is initially released from Rayleigh's mode $(n=2)$ described by the following equation: 


$$
R(\theta, t=0)=R_{0}\left(1+\varepsilon P_{2}(\cos (\theta))-\frac{1}{5} \varepsilon^{2}\right)
$$

where $P_{2}$ is the second degree Legendre polynomial and $\varepsilon=0.08$. The $3 \mathrm{D}$ mesh used here is composed of 194981 nodes and 1159517 elements.

Following the theoretical analysis in section 3, the natural frequency and the damping rate of the 3D iron droplet are :

$$
\left\{\begin{array}{c}
\omega_{2,0}=\sqrt{\frac{8 \gamma}{\rho^{l} R_{0}^{3}}} \\
\lambda_{2}=\frac{5 \mu^{l}}{\rho^{l} R_{0}^{2}}
\end{array}\right.
$$

$\omega_{2,0}=280.54$ rad.s $s^{-1}$ and $\lambda_{2}=0.46 \mathrm{~s}^{-1}$. So $\Delta_{2}^{\prime}=-78696 \mathrm{rad}^{2} . \mathrm{s}^{-2} \approx-\omega_{2,0}^{2}$ Therefore, the 3D iron droplet shows an underdamped regime. The analytical solution for this regime is:

$$
R(\theta, t)=R_{0}\left(1+\varepsilon_{2}(t) P_{2}(\cos (\theta))-\frac{1}{5} \varepsilon_{2}^{2}(t)\right)
$$

where

$$
\varepsilon_{2}(t) \approx \varepsilon e^{-\lambda_{2} t} \cos \left(\omega_{2,0} t\right)
$$

As in the previous sections, we assess the accuracy of the numerical solution by comparing it to the derived analytic solution. We extract from the simulation the time-evolution of the displacement of the upper point of intersection of the interface with the z-axis $(\theta=0)$. The comparison is shown in figure 8 .

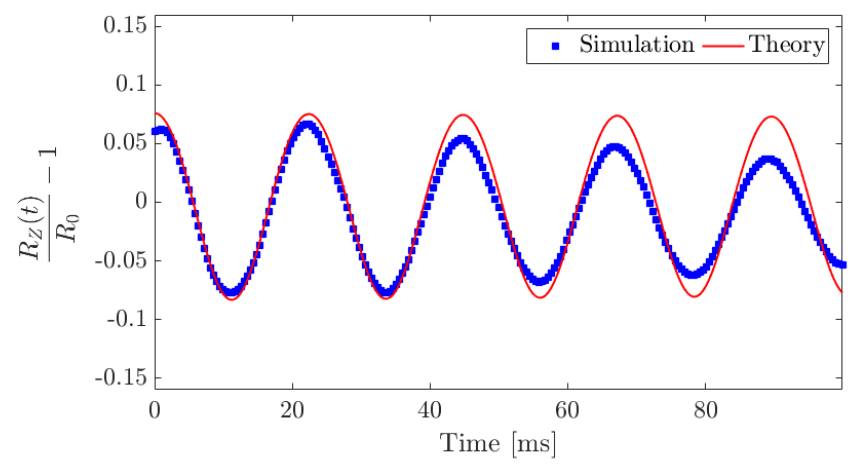

Figure 8: The time evolution of the displacement of the upper point of intersection of the interface with the z-axis

One can notice that the numerical signal is synchronised with the theoretical variations. It means that free surface dynamics driven by surface tension is well predicted. However, the amplitudes are more damped in the numerical simulation. The origin of this over-estimation of damping behaviour is probably attributed to numerical diffusion around the interface due to its thickness. In fact, in this present 3D simulation, the mesh resolution is coarser than the resolution of the $2 \mathrm{D}$ mesh which gave a very good estimation of the damping rate of the oscillations. Consequently, the thickness of the interface which depends on the mesh size is greater in the present 3D simulation than it must be. 
Overall, these results give confidence that the use of a convected Level-Set method combined with a Variational MultiScale method to solve the unsteady Navier-Stokes equations works well and could play an important role for modelling the behaviour of drops driven by surface tension. However, this numerical framework requires a high mesh resolution around the interface to reduce the numerical diffusion which adds an artificial damping to the simulated phenomenon. The use of a high-resolution mesh in 3D is very time-consuming. A dynamic conservative mesh adaptation seems a very promising tool to add to the present numerical framework for the purpose of increasing the accuracy with a reasonable computational time.

\section{Conclusions}

The development of an analytical and a numerical method to study the damped oscillations of a droplet are presented. The derivation of the theoretical solution in the frame of small-amplitude oscillations is detailed. The accuracy and the robustness of the proposed Eulerian framework to simulate in both $2 \mathrm{D}$ and 3D two-fluid flow with surface tension is thoroughly discussed. Simulations of different oscillations modes for a liquid iron droplet immersed in a very low density gas are presented. The obtained results and comparisons show that the two-fluid flow solver based on stabilised finite element method is able to exhibit good stability and accuracy properties. Further investigations will take into account the use of dynamic anisotropic mesh adaptation to increase accuracy and to reduce computational time. Application to experiments conducted with the Electromagnetic Levitator facility onboard the International Space Station are foreseen.

\section{Acknowledgements}

This work is supported by the European Space Agency (Noordwijk, NL) under the projects NEQUISOL (contract number 15236/02/NL/SH) and CCEMLCC (contract number 20277/06/NL/VJ).

\section{References}

[1] S. D. Hoath, W.-K. Hsiao, G. D. Martin, S. Jung, S. A. Butler, N. F. Morrison, O. G. Harlen, L. S. Yang, C. D. Bain, I. M. Hutchings, Oscillations of aqueous PEDOT:PSS fluid droplets and the properties of complex fluids in drop-on-demand inkjet printing, Journal of Non-Newtonian Fluid Mechanics 223 (2015) 28-36.

[2] N. Ashgriz, M. Movassat, Oscillation of Droplets and Bubbles, in: N. Ashgriz (Ed.), Handbook of Atomization and Sprays, chap. 5, Springer, Boston, MA, 125-144, 2011.

[3] M. Rappaz, D. Corrigan, L. A. BOAtner, Analysis of Ripple Fomartion in Single Crystal Spot Welds, Modeling of Casting, Welding and Advanced Solidification Processes 7 (1998) 713.

[4] A. J. Yule, J. J. Dunkley, Atomization of melts, in: Handbook of Atomization and Sprays, Clarendon press, Oxford, U. K., 1994.

[5] I. Egry, E. Ricci, R. Novakovic, S. Ozawa, Surface tension of liquid metals and alloys - Recent developments, Advances in Colloid and Interface Science 159 (2010) 198-212.

[6] H. J. J. Staat, A. van der Bos, M. van den Berg, H. Reinten, H. Wijshoff, M. Versluis, D. Lohse, Ultrafast imaging method to measure surface tension and viscosity of inkjet-printed droplets in flight, Experiments in Fluids 58:2.

[7] L. Rayleigh, On the Capillary Phenomena of jets, Proceedings of the Royal Society of London 29 (1879) 71-97.

[8] H. Lamb, Hydrodynamics, Cambridge University Press, sixth edn., 1932.

[9] H. Lamb, On the oscillations of a viscous spheroid, London Mathematical Society 13 (1881) 51-66.

[10] R. S. Valentine, N. F. Sather, W. J. Heideger, The motion of drops in viscous media, Chemical Engineering Science 20 (1965) 719-728.

[11] C. Miller, L. Scriven, The oscillations of a luid droplet immersed in another fluid, Journal of Fluid Mechanics 32 (1968) $417-435$.

[12] A. Prosperetti, Free oscillations of drops and bubbles : the initial-value problem, Journal of Fluid Mechanics 100 (1980) $333-347$.

[13] A. Prosperetti, Viscous Effects on perturbed spherical flows, Quarterly of Applied mathematics 34 (1977) $339-352$.

[14] J. Tsamopoulos, R. Brown, Nonlinear oscillations of inviscid drops and bubbles, Journal of Fluid Mechanics 127 (1983) 519-537.

[15] T. S. Lundgreen, N. N. Mansour, Oscillations of drops in zero gravity with weak viscous effects, Journal of Fluid Mechanics 194 (1988) 479-510. 
[16] E. Trinh, T. G. Wang, Large-amplitude free and driven drop-shape oscillations: experimental observations, Journal of Fluid Mechanics 122 (1982) 315-338.

[17] G. B. Foote, A numerical method for studying liquid drop behavior: simple oscillation, Journal of Computational Physics 11 (1973) 507-530.

[18] X. Xiao, R. W. Hyers, R. K. Wunderlich, H.-J. Fecht, D. M. Matson, Deformation induced frequency shifts of oscillating droplets during molten metal surface tension measurement, Applied Physics Letters 113(1) (2018) 011903.

[19] D. L. Cummings, D. A. Blackburn, Oscillations of magnetically levitated aspherical droplets, Journal of Fluid Mechanics 224 (1991) 395-416.

[20] A. Bratz, I. Egry, Surface oscillations of electromagnetically levitated viscous metal droplets, Journal of Fluid Mechanics 298 (1995) 341-359.

[21] R. K. Wunderlich, M. Mohr, Non-linear effects in the oscillating drop method for viscosity measurements, High Tempertures- High Pressures 48 (2019) 253-277.

[22] R. Scardovelli, S. Zaleski, Direct numerical simulation of free-surface and interfacial flow, Annual Review of Fluid Mechanics 31 (1999) 567-603.

[23] D. Joseph, Potential flow of viscous fluids: Historical notes, International Journal of Multiphase Flow 32 (2006) $285-310$.

[24] C. Bahbah, M. Khalloufi, A. Larcher, Y. Mesri, T. Coupez, R. Valette, E. Hachem, Conservative and adaptive level-set method for the simulation of two-fluid flows, Computers Fluids 191 (2019) 104223.

25] A. Smolianski, Numerical Modeling of Two-Fluid Interfacial Flows, Ph.D. thesis, University of Jyvskyl, 2001.

[26] J. U. Brackbill, D. B. Kothe, C. Zemach, A continuum method for modeling surface tension, Journal of Computional Physics 100 (1992) 335-354.

[27] T. Hughes, L. P. Franca, M. Balestra, A new finite element formulation for computational fluid dynamics: V. Circumventing the babuka-brezzi condition: a stable Petrov-Galerkin formulation of the stokes problem accommodating equal-order interpolations, Computer Methods in Applied Mechanics and Engineering 59 (1986) 85-99.

[28] T. Hughes, Multiscale phenomena: Greens functions, the Dirichlet-to-Neumann formulation, subgrid scale models, bubbles and the origins of stabilized methods, Computer Methods in Applied Mechanics and Engineering 127 (1995) 387-401.

[29] T. Hughes, G. Feijo, L. Mazzei, J.-B. Quincy, The variational multiscale methoda paradigm for computational mechanics, Computer Methods in Applied Mechanics and Engineering 166 (1998) 3-24.

[30] E. Hachem, B. Rivaux, T. Kloczko, H. Digonnet, T. Coupez, Stabilized finite element method for incompressible flows with high Reynolds number, Journal of Computational Physics 229 (2010) 8643-8665.

[31] R. Codina, Stabilization of incompressibility and convection through orthogonal sub-scales in finite element methods, Computer Methods in Applied Mechanics and Engineering 190 (2000) 1579-1599.

\section{Appendix A. Derivation of the energy balance}

To obtain the energy balance of the drop, we integrate the dot product of (1) and the velocity vector over the volume of $\Omega$ as follows:

$$
\iiint_{\Omega}\left[\rho\left(\frac{\partial \boldsymbol{u}}{\partial t}+\nabla \boldsymbol{u} \cdot \boldsymbol{u}\right)-\nabla \cdot \boldsymbol{\sigma}\right] \cdot \boldsymbol{u} d V=0
$$

By integrating by part the stress tensor term we can write:

$$
\iiint_{\Omega} \frac{1}{2} \rho\left(\frac{\partial \boldsymbol{u}^{2}}{\partial t}+\boldsymbol{u} \cdot \nabla \boldsymbol{u}^{2}\right) d V+\iiint_{\Omega} \boldsymbol{\sigma}: \nabla \boldsymbol{u} d V-\iint_{\Gamma} \boldsymbol{\sigma} \cdot \boldsymbol{n} \cdot \boldsymbol{u} d S=0
$$

Using the Reynolds transport formula and the fact that the $\boldsymbol{\nabla} \cdot \boldsymbol{u}=0$ we rewrite the first integral and we obtain the kinetic energy rate.

$$
\begin{aligned}
\iiint_{\Omega} \frac{1}{2} \rho\left(\frac{\partial \boldsymbol{u}^{2}}{\partial t}+\boldsymbol{u} \cdot \nabla \boldsymbol{u}^{2}\right) d V & =\frac{d}{d t} \iiint_{\Omega} \frac{1}{2} \rho \boldsymbol{u}^{2} d V \\
& =\frac{d E_{k i n}}{d t}
\end{aligned}
$$

We use the constitutive law of a Newtonian fluid and we show that the second integral represents the 
work of the viscous force.

$$
\begin{aligned}
\iiint_{\Omega} \boldsymbol{\sigma}: \nabla \boldsymbol{u} d V & =\iiint_{\Omega} 2 \mu \dot{\boldsymbol{\epsilon}}: \nabla \boldsymbol{u}-p \nabla \cdot \boldsymbol{u} d V \\
& =\iiint_{\Omega} 2 \mu \dot{\boldsymbol{\epsilon}}: \nabla \boldsymbol{u} d V=\iiint_{\Omega} 2 \mu \dot{\boldsymbol{\epsilon}}: \dot{\boldsymbol{\epsilon}} d V \\
& =\dot{W}_{\text {vis }}
\end{aligned}
$$

Considering the dynamic condition (5) at the interface $\Gamma$, the surface integral in (A.2) becomes:

$$
-\iint_{\Gamma} \boldsymbol{\sigma} \cdot \boldsymbol{n} \cdot \boldsymbol{u} d S=\iint_{\Gamma} \gamma \kappa \boldsymbol{u} \cdot \boldsymbol{n} d S
$$

Using the fact that $\kappa=-\nabla_{s} . \boldsymbol{n}$, the integration by parts over the closed surface $\Gamma$ gives:

$$
\iint_{\Gamma} \gamma \kappa \boldsymbol{u} \cdot \boldsymbol{n} d S=\iint_{\Gamma} \nabla_{\boldsymbol{s}} \cdot(\gamma \boldsymbol{u}) d S
$$

Finally, we use the Leibniz integral rule for a moving surface and we obtain the potential energy rate:

$$
\begin{aligned}
-\iint_{\Gamma} \boldsymbol{\sigma} \cdot \boldsymbol{n . u} d S & =\iint_{\Gamma} \frac{\partial \not}{\partial t}+\nabla_{\boldsymbol{s}} \cdot(\gamma \boldsymbol{u}) d S \\
& =\frac{d}{d t} \iint_{\Gamma} \gamma d S \\
& =\frac{d E_{p o t}}{d t}
\end{aligned}
$$

